\title{
Recursive Measures of Edge Accuracy on Digital Images
}

\author{
Jeffrey Zheng and Chris Zheng
}

\begin{abstract}
In this chapter, an edge accuracy model is proposed on digital images and five types of edge detection methods are discussed as examples to investigate their edge maps undertaken recursive operations. Using invariant criterion, it is possible to compare different schemes in accuracy, consistency, completeness and simplicity. This provides general mechanism in relation to accurate edge extractions from digital images.
\end{abstract}

Keywords Edge detection $\cdot$ Accuracy $\cdot$ Invariant $\cdot$ Digital image

\section{Introduction}

Edge detection plays a fundamental importance in image analysis, processing and computer vision applications. As the first step of visual perception, extensive R\&D has being focused for 40 years (more than forty thousand years-drawing arts in human civilization). Many useful edge detection operators have been invented and applied in wider applications.

From an operational viewpoint, edge detection creates edge maps from images shown in Fig. 1a. Edge detection operators identify significant changes from visual objects as their edges or contours. From a historical viewpoint, common edge detec-

This work was supported by Australian Commercialising Emerging Technologies, (COMET) program.

J. Zheng $(\varangle)$

Key Laboratory of Software Engineering of Yunnan, Yunnan University, Kunming, Yunnan, China e-mail: conjugatelogic@yahoo.com

C. Zheng

Tahto, Sydney, Australia

e-mail: z@caudate.me

(C) The Author(s) 2019

J. Zheng (ed.), Variant Construction from Theoretical Foundation to Applications,

https://doi.org/10.1007/978-981-13-2282-2_12 


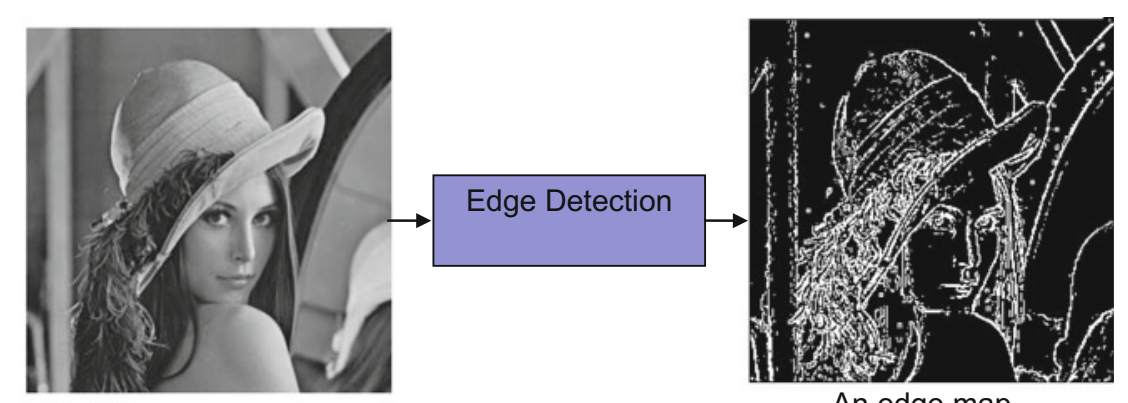

An image

(a)

An edge map

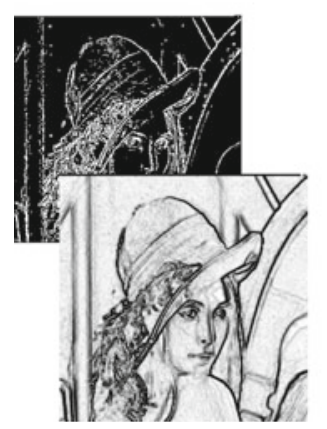

Extracted edge maps

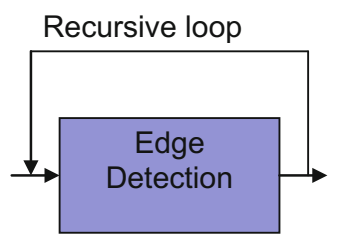

(b)

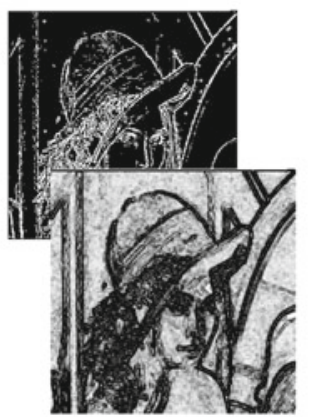

Recursive edge maps

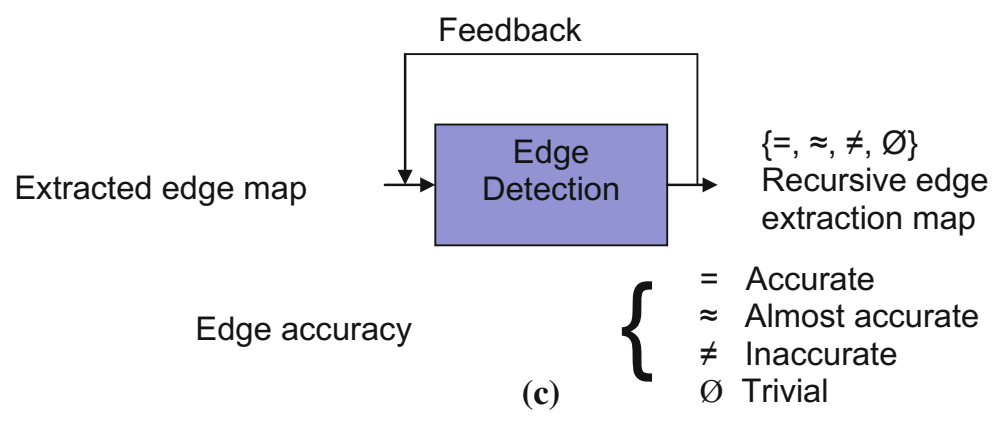

Fig. 1 Recursive edge extraction. a Edge detection; b Recursive edge maps; c Edge accuracy measures

tion approaches are divided into five approaches. Traditional edge detections have three main categories: Gradient, Laplacian and Gaussian; another two categories are mathematical morphology and conjugate. The five categories will be briefly introduced as follows. 


\subsection{Gradient}

Gradient scheme has a direction corresponding to convolution operations; we can use $2 \times 2,3 \times 3$ matrices or more complicated schemes to construct relevant operators, for example, Roberts operator uses $2 \times 2$ matrix to detect edges on main diagonal or anti-diagonal directions. Prewitt, Sobel and Isotropic schemes take $3 \times 3$ matrices using different parameters to extract horizontal or vertical edges from digital images shown in Fig. 2a.

\subsection{Laplacian}

A typical Laplacian scheme is Marrs-Hildreth's zero crossings. This scheme uses the second differential information to determine zero crossings of the edges shown in Fig. 2b.

\subsection{Gaussian}

Canny edge filter plays a significant role in advanced edge detection applications from late of 1980s. This scheme applies Gaussian smoothing filter first, then gradient operations and finally thinning processes and its final results shown in Fig. 2c. Different from Gradient and Laplacian schemes, Canny edge detection provides controllable parameters to balance noise levels and significant edge components. Because of its controllable properties, this scheme widely used in many practical applications in relation to significant edge components.

\subsection{Mathematical Morphology}

Mathematical morphology plays an important role in advanced image analysis and processing applications from 1980s. Using discrete patterns as morphological masks, the method applies erosion and dilation, opening and closing operations on the processed images. This method distinguishes edge and non-edge masks. In general, only translation invariant can be retained in operations. Each time of basic operation uses one mask on either erosion or dilation corresponding to reduce or extend boundaries of the visual objects. There is no simple relationship between the selected mask states and edge states. Two edge maps using a crossing mask under either erosion or dilation are shown in Fig. 2d. Each edge map has been calculated by either dilated or eroded output image subtracted by the input edge map. 


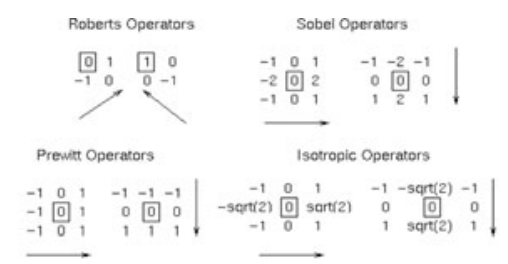

(a) Gradient

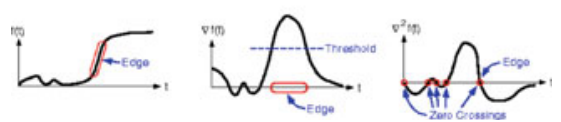

(b) Laplacian (Zero crossing)
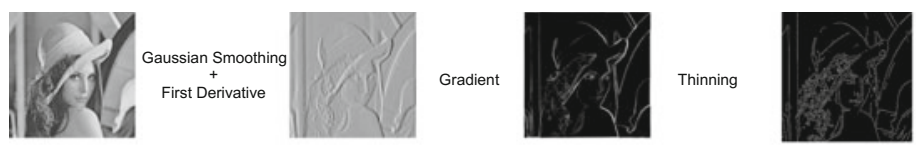

(c) Gaussian (Canny)

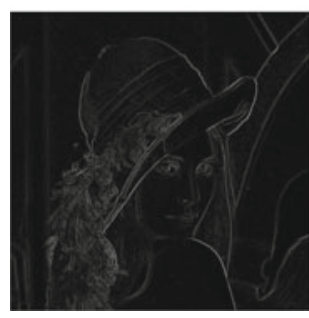

Erosion Edge Map

(d) Mathematical Morphology

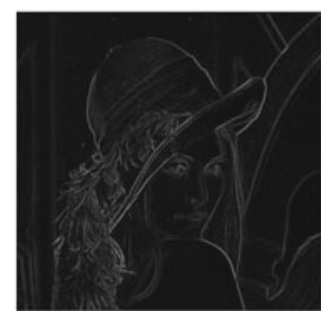

Dilation Edge Map

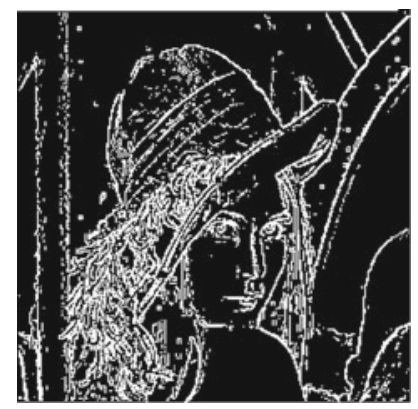

Negative map:

Lena.NM.50.50.10.bABCDEFGHIJKLac.-4.256.256.gif

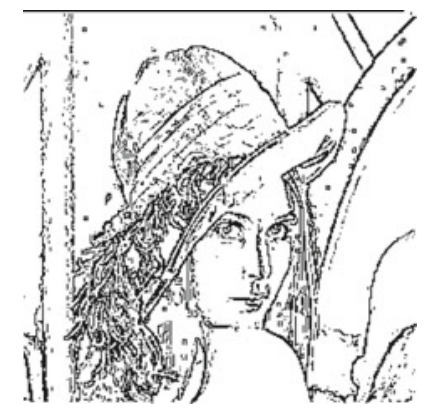

Positive map:

Lena.PM.50.50.10.bABCDEFGHIJKLac. -4.256 .256$. gif

(e) Conjugate (Negative \& Positive maps)

Fig. 2 Different edge detection methods. a Gradient; b Laplacian; c Gaussian; d Mathematical morphology; e Conjugate 


\subsection{Conjugate}

Conjugate scheme has been developed from 1990s and based on a full pattern classification of nearest neighbourhood relationship of discrete states on regular plan lattices under rotation, reflection and translation invariants. This approach can express local patterns into invariant groups such as isolated, inner, block edge and intersection to organise whole pattern space as a hierarchical construction. Both background and foreground information need to be represented as balanced structures in conjugate phase space. Under certain conditions, it is feasible to use two types of edge maps in representations. In Fig. 2e, two typical edge maps are illustrated to use conjugate scheme:

- Negative (White edge map on black background) and

- Positive (Black edge map on white background).

From edge detection considerations, different operations provide special properties to be emphasised by various visual information from images. Simple convolution filters may provide fast process; however, it is highly possible to be sensitively influenced by minor noise levels. Among three traditional edge detection schemes, Canny edge detector shows an important characteristic with a series of controllable edge maps in reliable properties. Because distinct edge detectors have different behaviours, it is very hard for applicants to make simple selections apply the best one among schemes. Mathematical morphology applies discrete masks in operations. Since edge maps normally do not correspond to masks themselves directly, it is difficult to establish a link from relevant operations and edge detection results.

Considering edge detection operation extracts edge map from digital images. Under this viewpoint, we need to establish a proper model in determining invariant properties among edge detection schemes.

\section{Recursive Model of Edge Accuracy}

Different edge detection methods cover various applications with advantages in many aspects. From a practical viewpoint, it is hard for users to make proper judgment on which method provides the best edge map to satisfy suitable applications. From history of edge detection research, no model can provide general mechanism in systematic comparison among distinguished methods. Since the target of different edge detections creates edge maps, it is natural for us to determine under which conditions the edge maps can represent true edge. 


\subsection{Question}

Could an extracted edge map be a true edge representation?

From a morphological viewpoint, true edge map needs to have invariant properties relevant to their geometric and topological constrains. In many theories and practices in relation to dynamic systems and cybernetics, recursive methods and models have been approved to be a foundational importance in detailed analysis tasks. A recursive model has been applied in testing edge detection operators to explore their refined properties shown in Fig. 1b. Using this feedback mechanism, edge map needs to be looped back again undertaken the same type of edge detection operators. The recursive loop shows an important magnification to identify dynamic behaviours among input and output pairs directly.

\section{Four Types of Edge Accuracy Measures}

Under the recursive approach, a true edge representation must be the recursive edge map itself. Such invariant of recursive operations can be observed as intrinsic properties in relation to the edge detection operators themselves. In addition to invariant properties, many rich effects among input and output pairs need to be concerned. To make proper judgment among recursive results, it is essential to apply four different accurate measures shown in Fig. 1c. They are $\{=, \approx, \neq, \varnothing\}$ representing accurate, almost accurate, inaccurate and trivial behaviours, respectively, between input and output edge maps. From matched results between extracted edge map and its recursive edge extraction map, it is feasible to determine the category in which generated results need to be belonging to. This provides a general model independent of a specific edge detection scheme. If anyone would like to check which category could be belonging to a special scheme, the person can simply apply this recursive mechanism to check specific method itself directly in explorations.

\section{Four Sample Groups of Recursive Edge Maps}

In Fig. 3a-d, four groups of recursive edge maps are generated in illustration. Two operators are selected from Photoshop: Find edge (Gradient) Fig. 3a and trace contour (Zero crossings) Fig. 3b. Find edge operation has a clear variant property, and trace contour will have a flip-flop behaviour after certain operations. One example is selected from Canny edge detector shown in Fig. 3c. Recursive results of Canny operation show that two sets of examples are shown in Fig. $3 \mathrm{~d}$ for mathematical morphology. It is interesting to see dilation representing almost invariant properties and erosion creating edge map similar to zero crossing effects. To show different recursive properties of conjugate scheme, four sub-operators are illustrated in Fig. 3e1-e4. 
Table 1 Edge detection schemes and their accuracy properties

\begin{tabular}{l|l|l|l|l}
\hline Operator & Edge quality & Noise sensitivity & Accuracy & Recursive maps \\
\hline Find edge & Good, fair & Very high & \pm 2 pixels & $\neq, \varnothing$ \\
\hline Trace contour & Better, good, fair & High & \pm 1 pixels & $\neq, \approx$ \\
\hline Canny edge & Better, good, fair & Controllable & \pm 2 pixels & $\neq$ \\
\hline $\begin{array}{l}\text { Mathematical } \\
\text { morphology }\end{array}$ & Better, good, fair & High & \pm 1 pixels & $\approx, \neq, \varnothing$ \\
\hline Conjugate map & $\begin{array}{l}\text { Best, better, } \\
\text { good, fair }\end{array}$ & Full controllable & $\begin{array}{l}\leq 1 \text { pixel } \\
\text { True edge }\end{array}$ & $=, \approx, \neq, \varnothing$ \\
\hline
\end{tabular}

Each group shows a specific category among three non-trivial results. In conjugate edge detection operators, there are two types of controllable parameters that are available corresponding to meta-shape parameters $\{\mathrm{A}, \ldots, \mathrm{L}, \mathrm{a}, \ldots, 1\}$ and enhanced ratio control $\{-8, \ldots, 8\}$. Both controllable parameters can provide universal edge representation on true edge map to support various edge representations undertaken selected operations.

\section{Comparison}

Using the five categories, it is feasible to make summary in Table 1. This provides a systematic way in comparison. 


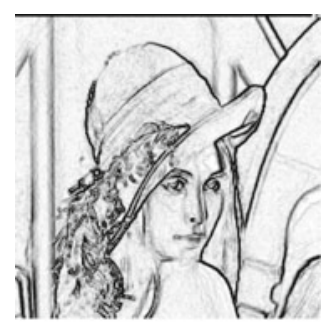

(A1) The first edge map

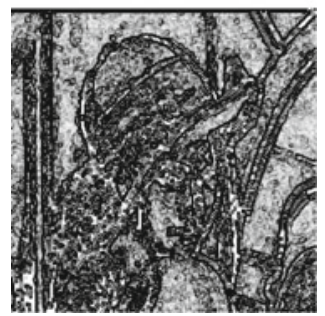

(A3) The third edge map

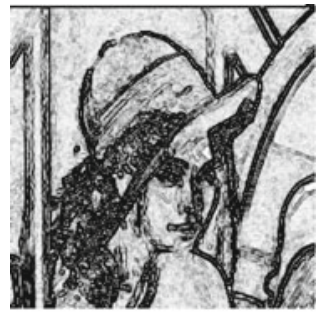

(A2) The second edge map

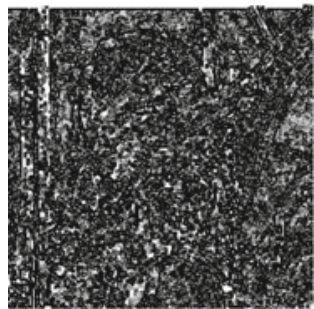

(A4) The fourth edge map

$(\mathrm{A} 1) \neq(\mathrm{A} 2) \neq(\mathrm{A} 3) \neq(\mathrm{A} 4) \quad$ No invariant edge map available!

Recursive condition: Directly use find edges filter to each map

(A). Photoshop: Find Edges (Gradient)

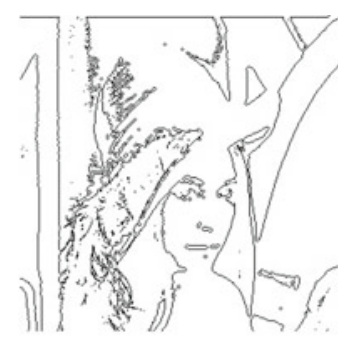

(B1) The first map

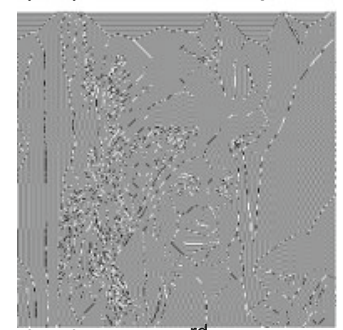

(B3) The $53^{\text {rd }}$ map

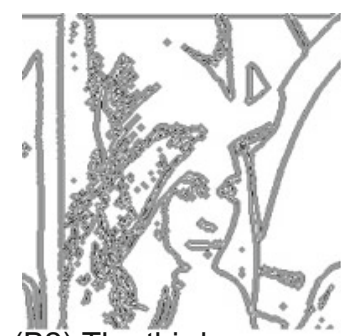

(B2) The third map

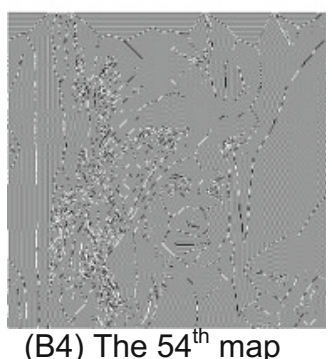

$(B 1) \neq(B 2) \neq(B 3) \approx(B 4) \quad$ Flip flap variations after the $53^{\text {rd }}$ operation

Recursive condition: Trace contour filter (level $=119$, edge $=$ low)

(B). Photoshop: Trace Contour (Zero Crossing)

Fig. 3 Recursive maps of different edge detection operators. a Find edges; b Trace edge; c Canny edge detection; $\mathbf{d}$ Morphology; e Conjugate edge detection 


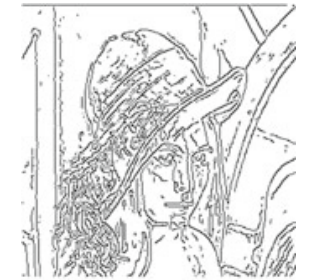

(C1) The first edge map

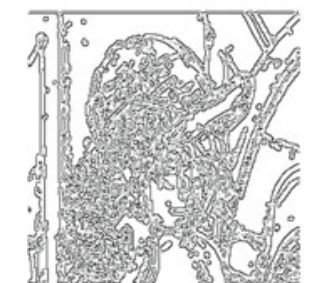

(C3) The third edge map

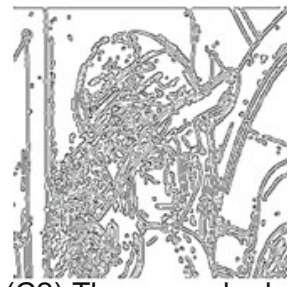

(C2) The second edge map

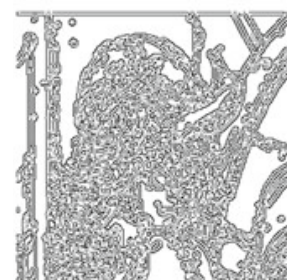

(C4) The fourth edge map

$(C 1) \neq(C 2) \neq(C 3) \neq(C 4) \quad$ No invariant edge map available!

Recursive condition: Sigma $=1$, high threshold $=8$, low threshold $=7$

(C). Canny Edge Detection (Gaussian smooth + Gradient + Thinning)

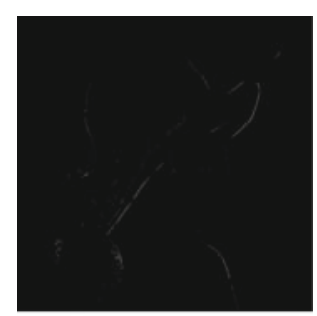

(D11) The first edge map

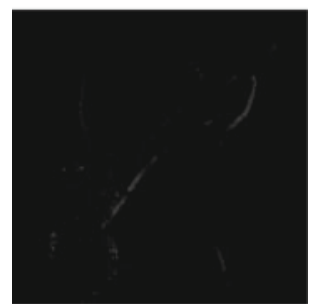

(D13) The third edge map

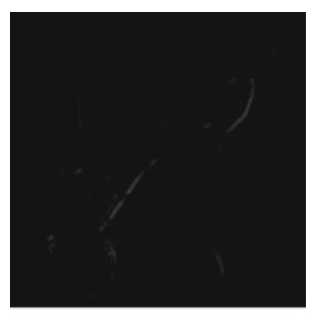

(D12) The second edge map

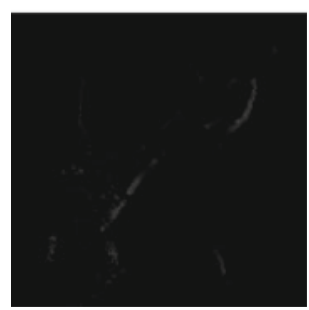

(D14) The $4^{\text {th }}$ edge map

$(D 11) \neq(D 12) \neq(D 13) \neq(D 14)$ Edge maps invariant Recursive Condition: Erosion using a crossing mask

Fig. 3 (continued) 


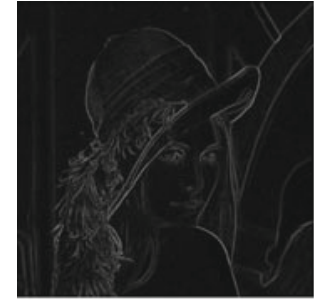

(D21) The first edge map

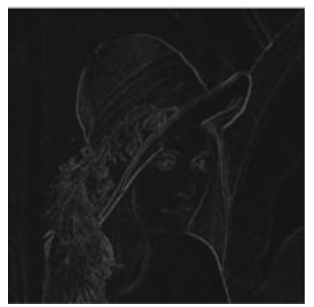

(D23) The third edge map

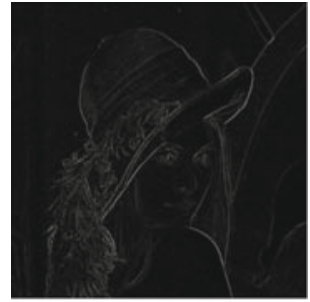

(D22) The second edge map

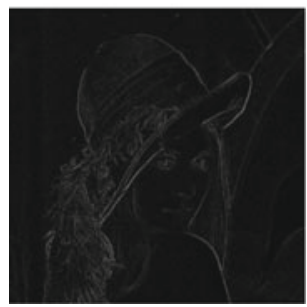

(D24) The $4^{\text {th }}$ edge map

$(D 11) \approx(D 12) \approx(D 13) \approx(D 14)$ Edge maps almost invariant Recursive Condition: Dilation using a crossing mask

(D). Mathematical Morphology
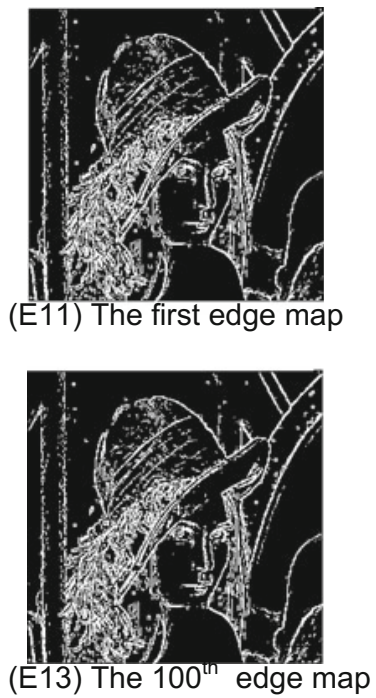

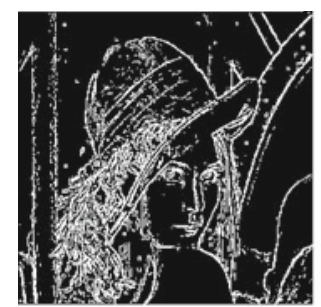

(E12) The fifth edge map

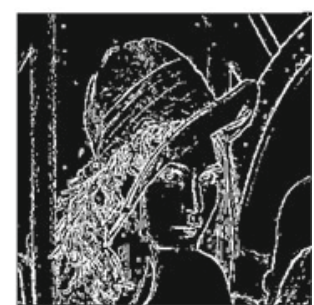

(E14) The $1000^{\text {th }}$ edge map

$(E 11)=(E 12)=(E 13)=(E 14)$ Edge maps invariant Recursive Condition: NM 505010 abcdefghijkl -2

Fig. 3 (continued) 

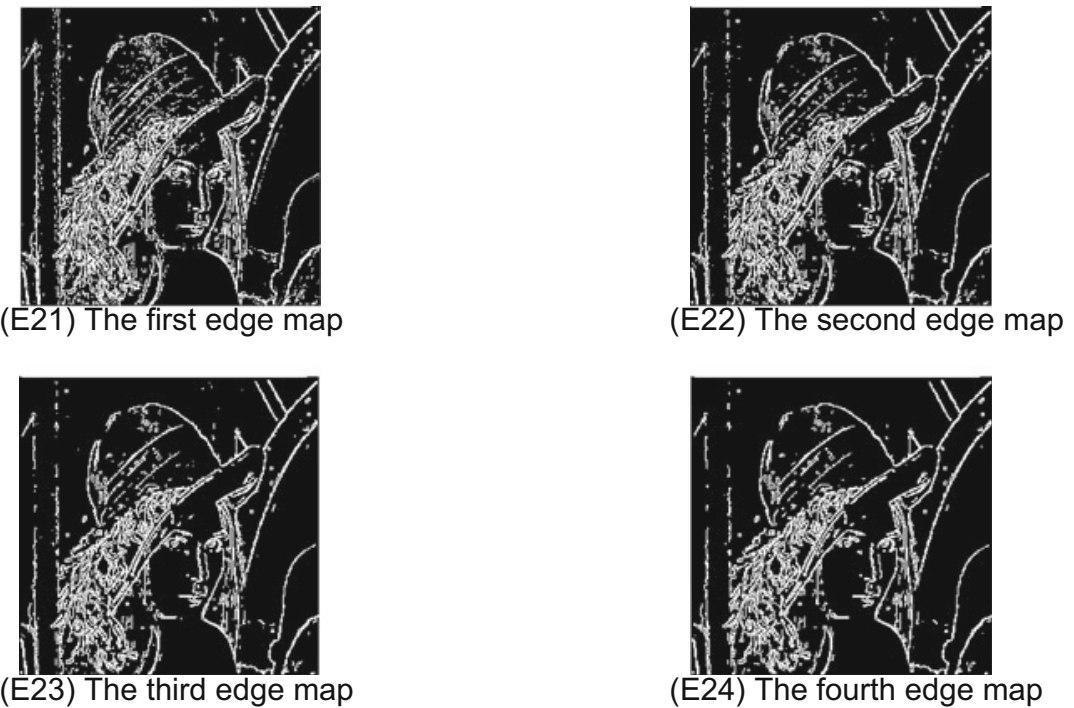

$(E 21) \approx(E 22) \approx(E 23) \approx(E 24) \quad$ Similar edge maps with noise removing Recursive Condition: NM.50.50.10.abcdefghijkIABC.-2
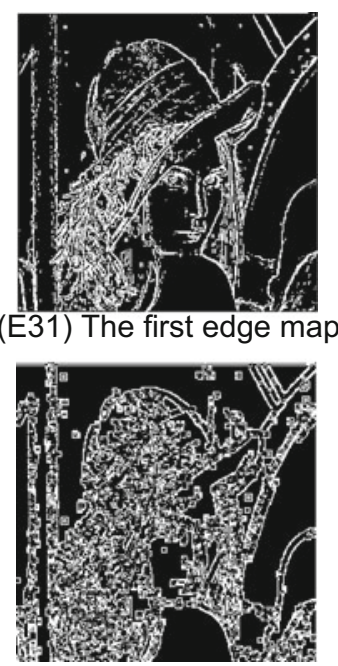

(E33) The third edge map

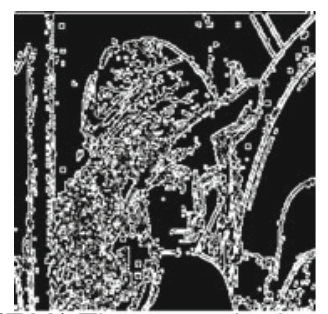

(E32) The second edge map

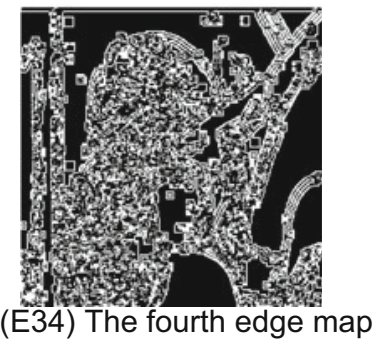

$(E 31) \neq(E 32) \neq(E 33) \neq(E 34) \quad$ Changed edge maps

Recursive Condition: PM.50.50.10. cdefCDEF.-2

Fig. 3 (continued) 


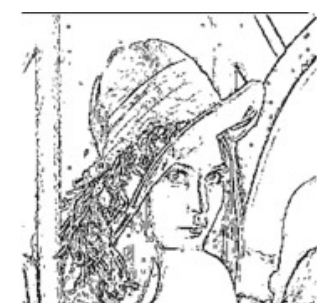

(E41) The first edge map

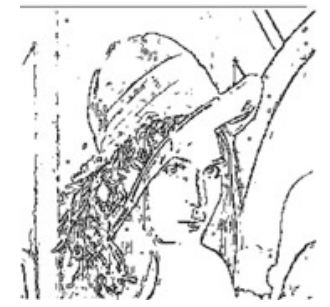

(E43) The third edge map

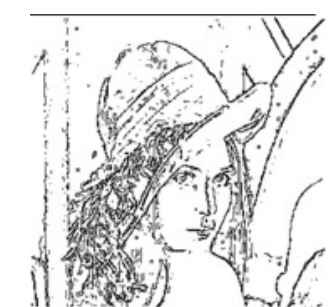

(E42) The second edge map

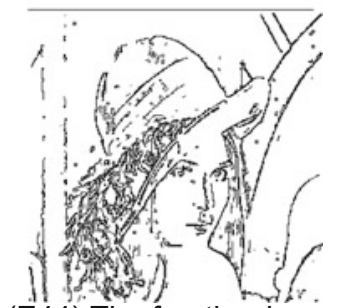

(E44) The fourth edge map

$(E 41) \approx(E 42) \approx(E 43) \approx(E 44) \quad$ Similar edge maps with noise removing Recursive Condition: PM.50.50.10.ABCDEFGHIJKLabc.-2

(E). Conjugate Edge Detection

Fig. 3 (continued) 


\section{Conclusion}

Existing edge detections are without unique recursive maps as their representations. Conjugate technology provides full controls to create true edge maps in accuracy and invariance.

True edge maps contain unique shape information in fundamental importance to support all visual applications.

Open Access This chapter is licensed under the terms of the Creative Commons Attribution 4.0 International License (http://creativecommons.org/licenses/by/4.0/), which permits use, sharing, adaptation, distribution and reproduction in any medium or format, as long as you give appropriate credit to the original author(s) and the source, provide a link to the Creative Commons license and indicate if changes were made.

The images or other third party material in this chapter are included in the chapter's Creative Commons license, unless indicated otherwise in a credit line to the material. If material is not included in the chapter's Creative Commons license and your intended use is not permitted by statutory regulation or exceeds the permitted use, you will need to obtain permission directly from the copyright holder.

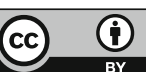

